\title{
Searching the Allais effect during the total sun eclipse of 11 July 2010
}

\author{
Horacio R. Salva* \\ Centro Atómico Bariloche and Instituto Balseiro, CNEA-UNCuyo-Conicet, Av. Bustillo 9500, (8400) Bariloche, Argentina
}

(Received 28 December 2010; published 16 March 2011)

\begin{abstract}
I have measured the precession change of the oscillation plane with an automated Foucault pendulum and found no evidence (within the measurement error) of the Allais effect. The precession speed was registered and, due the variations involved, if the precession speed would changed 0.3 degree per hour (increasing or decreasing the angle of the normal precession speed) during the all eclipse, it would be notice in this measurement.
\end{abstract}

DOI: 10.1103/PhysRevD.83.067302

PACS numbers: 95.10.Gi, 04.20.Cv, 45.50.Pk

\section{INTRODUCTION}

Maurice Allais had found in 1954, during the total sun eclipse of 30 June 1954 [1], an anomaly in the precession of the azimuth of his paraconical pendulum in a marathon measurement of several days, before and after the eclipse took place. He repeated his measurements in the next total sun eclipse of 2 October 1959 [2] and found a similar behavior. The magnitude of the eclipse at the measurement site (mems) was $\sim 0.4$. In the first case he observed that, after the eclipse began, the azimuth angle increased 13 degrees while the Foucault precession was -11.35 degrees per hour (measured in Saint-Germain-en-Laye, France, latitude $48.898^{\circ} \mathrm{N}$, mems $\sim 0.7$ ). At the end of the eclipse the azimuth was nearly at a value that it would be if the anomaly were not present. Following these events, many people tried to find any anomaly during the total sun eclipses. In 1970 Saxl and Allen [3] found a change in the period of a torsion pendulum, that they attributed to the same origin as the anomaly found by M. Allais on his pendulum. Nevertheless, T. Kuusela [4] and L. Jun et al. [5] measured the period of a torsion pendulum during the solar eclipse of 22 July 1990 and found no effect on the period during the eclipse. NASA organized a project of world measurement of the anomalies of the solar eclipse of 11 August 1999 [6]. There were measurements with Foucault pendulums, spring and superconducting gravimeters, seismographs, and so on.

This project is not finished yet, nevertheless some results are available. I. Mihaila et al. [7] measured the solar eclipse with a $14.21 \mathrm{~m}$ Foucault pendulum placed at The Mathematical Faculty of the Bucarest University. They found a difference of $1.8^{\circ}$ maximum between the azimuth of the pendulum with and without the eclipse (mems 0.95). They measured again the effect on the annular eclipse of 31 May 2003 [8] (mems 0.75). Recently, two reviews were published on the measurements and possible explanations of the Allais effect $[9,10]$.

*salva@cab.cnea.gov.ar

\section{THE APARATUS}

The pendulum was already described in [11]. It is a $12.5 \mathrm{~kg}$ lead bob hanging from a fixed support with a $4.975 \mathrm{~m}$ piano chord. It has a feedback amplifier that keeps continuous oscillation of the pendulum at $1^{\circ}$ amplitude. It also has an electromagnetic brake which keeps the elliptic trajectory in acceptable values (precession speed no higher than $0.1^{\circ} / \mathrm{h}$ ). There is a following system composed of two Hall sensors, which measures continuously the position of the plane of oscillation, the minor diameter, and the sense of rotation of the ellipse. The total precession is calculated as the derivative of the precession angle vs time with the last number of oscillations. Finally, the total precession measured is corrected by the ellipse precession.

\section{THE MEASUREMENTS}

At Bariloche, the total sun eclipse was a partial one, with magnitude 0.822. The data for Bariloche, taken from the NASA eclipse web site [12] were the following:

\begin{tabular}{lllll} 
& UT (h:m:s) & LT & Altitude & Azimuth \\
\hline Start & $19: 52: 22$ & $16: 52: 22$ & $13.5^{\circ}$ & $317.5^{\circ}$ \\
Max & $20: 57: 14$ & $17: 57: 14$ & $4.4^{\circ}$ & $305.3^{\circ}$ \\
End & $21: 56: 34$ & $18: 56: 34$ & $-5.1^{\circ}$ & $295.3^{\circ}$
\end{tabular}

The local time (LT) in Argentina is $3 \mathrm{~h}$ less than the universal time (UT). The azimuth given by NASA follows the convention: zero pointing north and increasing toward the east.

Bariloche was very far from the center of the total eclipse (nearly $1000 \mathrm{~km}$ ) and very near the end of it (similar longitude of Calafate, Santa Cruz, Argentina) (magnitude: 0.822). This eclipse took place in a region where there were still replications of the major earthquake of 27 February 2010 in Maule, Chile (magnitude 8.8). I had the chance of seeing several earthquakes in the hours following the eclipse. This shows the characteristic perturbation in the precession movement of the Foucault pendulum when an earthquake happens. It can be compared with the precession movement during the eclipse. The long time measurement taken during three weeks is shown in Fig. 1. 


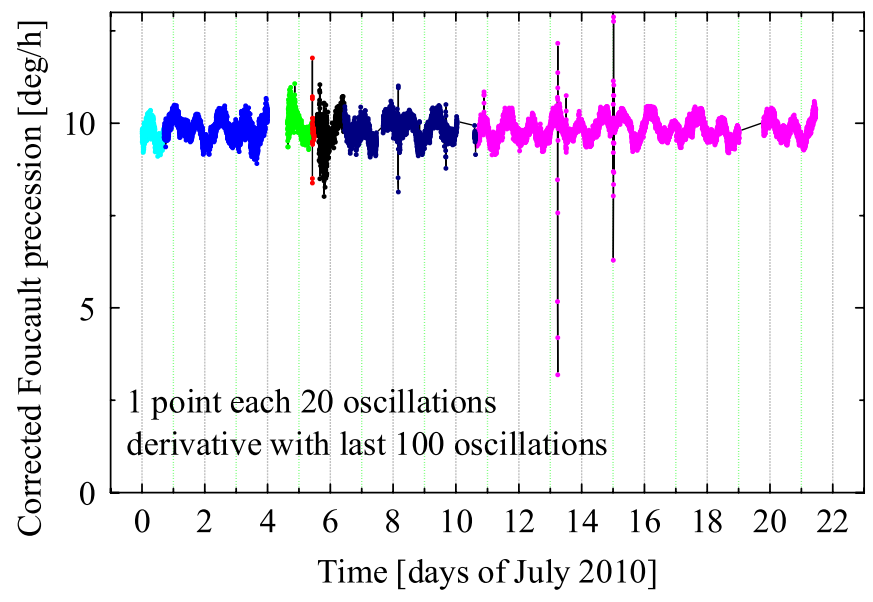

FIG. 1 (color online). Long time measurement during three weeks.

There are some hours when there were no measurement points. These correspond to situations when the following system "lost" the oscillation plane movement for several reasons (energy lost, program halted, etc.)

There are two kinds of noise, one with a long period (nearly $1 \mathrm{~d}$ ) and the other with a short time period. The second is due to the few motor pulses done in following the precession in one oscillation, and can be reduced by increasing the number of oscillations when calculating the precession speed. The long period noise (about 1.28 cycles/ day, as one referee pointed out) is probably a misalignment of some parts of the pendulum, because it has nearly the half time that the oscillation plane needs to precess $360^{\circ}$. As can be seen, there are some disturbances which belong to earthquakes. If Fig. 1 is expanded to a few days after the eclipse we have Fig. 2. There we have pointed the time when the eclipse started, its maximum, and the end. Some earthquakes are also seen and are numerated in the following (where universal time is given as year month day hours: minutes:seconds):

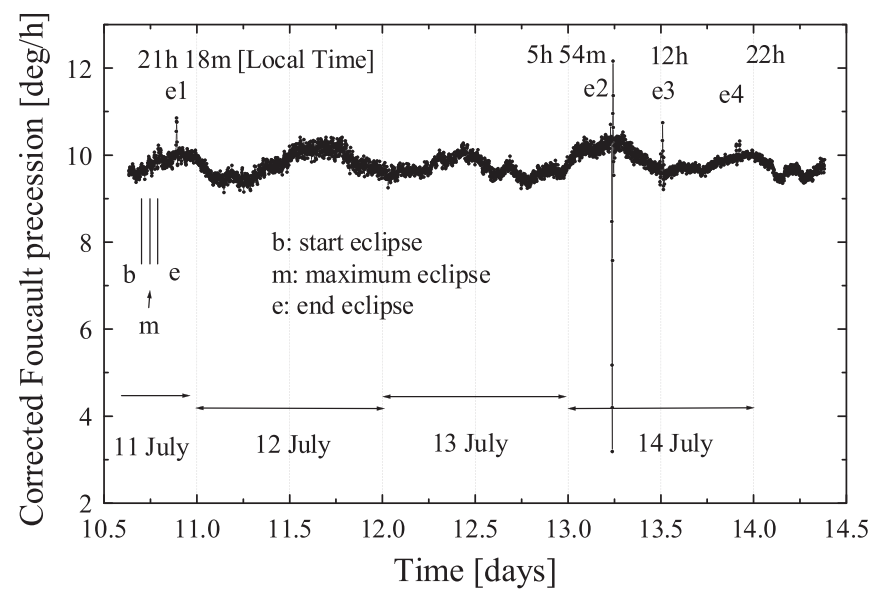

FIG. 2. Expanded time measurement.

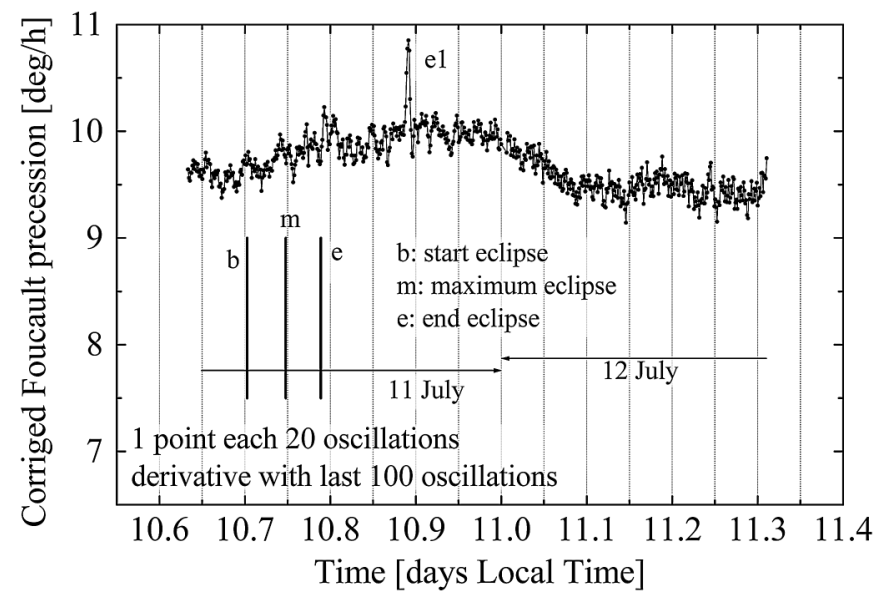

FIG. 3. Plot of measurements during the eclipse.

e1: Antofagasta, Chile, magnitude: 6.3, distance: 2125 km, UT: 20100712 00:11:21;

e2: Bio-Bio, Chile, magnitude: 5.3 , distance: $382 \mathrm{~km}$, UT: 20100714 08:21:13;

e2: Bio-Bio, Chile, magnitude: 6.6, distance: $381 \mathrm{~km}$, UT: 20100714 08:32:23;

e3: Bio-Bio, Chile, magnitude: 5.8, distance: $356 \mathrm{~km}$, UT: 201007 14 15:05:50;

e4: Offshore, Libertador O'Higgins, Chile, magnitude: 5.1, distance: 776 km, UT: 20100715 00:36:02;

e4: Offshore, Bio-Bio, Chile, magnitude: 5.0, distance: 406 km, UT: 201007 15 01:02:02.

These data were taken from the United States Geological Survey web page [13]. Figure 3 shows a more detailed plot of the measurements during the eclipse.

From the measurements and the time when the eclipse happened, we see that the pendulum did not move significantly from the short-term noise. If we define the sensibility of the measurement as the least signal that equals the short-term noise $\left(0.3^{\circ} / \mathrm{h}\right)$ the pendulum should change its precession movement less than $0.3^{\circ}$ between the beginning and the maximum of the eclipse. The azimuth is defined as the zero pointing south and increasing toward the west. The azimuth of the oscillation plane of the pendulum is the perpendicular direction to the plane. The total sun eclipse started with the pendulum oscillating at $211.9^{\circ}$, passed the maximum at $201.45^{\circ}$, and ended at $191.83^{\circ}$. I. Mihaila et al. [8] had found that the azimuth of oscillation was irrelevant relative to the azimuth of the sun during the eclipse.

\section{CONCLUSIONS}

The maximum deflection of the precession of the Foucault pendulum found in these measurements is very far from those obtained by M. Allais [1] $\left(13^{\circ}\right)$ and I. Mihaila [7,8] $\left(1.8^{\circ}\right)$ in other total sun eclipses. I saw nothing out of the noise, that is, the effect would be less than $0.3^{\circ}$ in $1 \mathrm{~h}$. We calculate the derivative of the 
precession of the last 100 oscillations; this means the last $7.5 \mathrm{~min}$. This fact is not a problem because, if there would be a change in the speed of precession of $0.3^{\circ}$ in the last 7.5 min, it surely would detected by the pendulum, as the earthquakes are. Even though certain structure is seen in the noise during the eclipse, it is not enough to assure that the effect is present. I believe that our place is not a favorable one to detect the effect, since we are very far from the maximum of the eclipse (in the middle of the Pacific Ocean) and near the end of it.
[1] M. Allais, Comptes Rendus de l'Académie des Sciences (France) 245, 2001 (1957).

[2] M. F. C. Allais, Aerosp. Eng. 18, 42 (1959); 18, 51 (1959); 18, 55 (1959).

[3] E. J. Saxl and M. Allen, Phys. Rev. D 3, 823 (1971).

[4] T. Kuusela, Phys. Rev. D 43, 2041 (1991).

[5] L. Jun, L. Jianguo, Z. Xuerong, V. Liakhovets, M. Lomonosov, and A. Ragyn, Phys. Rev. D 44, 2611 (1991).

[6] NASA Science, Decrypting the Eclipse, http://science.nasa.gov/science-news/science-at-nasa/1999/ast06aug99_1/; NASA Science, French Nobel Laureate Turns Back Clock, http://science.nasa.gov/science-news/science-at-nasa/1999/ ast12oct99_1/.
[7] I. Mihaila, N. Marcov, V. Pambuccian, and M. Agop, Proc. Rom. Acad., Ser. A 4, 1 (2003).

[8] I. Mihaila, N. Marcov, V. Pambuccian, and O. Racoveanu, Proc. Rom. Acad., Ser. A 5, 1 (2004).

[9] C. Duif, arXiv:gr-qc/0408023.

[10] X. Amador, J. Phys. Conf. Ser. 24, 247 (2005).

[11] H. R. Salva, R.E. Benavides, J.C. Perez, and D. J. Cuscueta, Rev. Sci. Instrum. 81, 115102 (2010).

[12] NASA, Eclipse Web Site, http://eclipse.gsfc.nasa.gov/ SEgoogle/SEgoogle2001/SE2010Jul11Tgoogle2.html.

[13] USGS, National Earthquake Information Center, http:// neic.usgs.gov/neis/bulletin/bulletin_esp.html. 